\title{
Underwater image processing and analysis: A review
}

\author{
Muwei Jian ${ }^{1,2,3^{*}}$, Xiangyu Liu ${ }^{l}$, Hanjiang Luo ${ }^{4}$, Xiangwei Lu $u^{1}$, Hui Y $u^{3^{*}}$, Junyu Dong ${ }^{5}$ \\ ${ }^{1}$ School of Computer Science and Technology, \\ Shandong University of Finance and Economics, Jinan, China. \\ ${ }^{2}$ School of Information Science and Engineering, Linyi University, Linyi, China. \\ ${ }^{3}$ School of Creative Technologies, University of Portsmouth, Portsmouth, UK. \\ ${ }^{4}$ School of Computer Science and Technology, \\ Shandong University of Science and Technology, Qingdao, China. \\ ${ }^{5}$ Department of Computer Science and Technology, Ocean University of China, Qingdao, China. \\ *(Co-correspondence author) E-mail: jianmuweihk@163.com; hui.yu@port.ac.uk
}

\begin{abstract}
With increasing attentions being drawn to the underwater observation and utilization of marine resources in recent years, underwater image processing and analysis have become an active research hotspot. Different from the general images, marine environment is usually faced with some complicated situations such as underwater turbulence and diffusion, severe absorption and scattering of water body, various noises, low contrast, uniform illumination, monotonous colour, complex underwater-background. In response to these typical challenges, a large body of works in underwater image processing has been exploited in recent years. This survey introduces a review of existing relatively mature and representative underwater image processing models, which are classified into seven categories including enhancement, fog removal, noise reduction, segmentation, salient object detection, colour constancy and restoration. We then objectively evaluate the current situations and future development trend of underwater image processing, and provide some insights into the prospective research directions to promote the development of underwater vision and beyond.
\end{abstract}

Keywords: underwater image, marine environment, underwater saliency detection, colour constancy

\section{Introduction}

Ocean occupies for 70 percent of the earth and contains rich natural resources, which has been a long-term and enduring concern for human development. With the rapid progresses of the marine exploitation and observation, there is increasing interests in the exploring this mysterious area of the ocean. However, confronted with relatively hash and unconstrained marine scenes, many severe adverse factors, include water with high turbidity, uneven illumination, monotonous color, complicated underwater-background, which seriously affect the integrity and availability of underwater image in practical application.

In order to tackle with these issues, many relevant underwater-vision researches have been conducted on exploring mature and effective underwater image processing (UIP) models in recent decades. In this review, we will discuss the processing and analyzing methods of underwater images, containing both traditional methods and recently emerged models of deep-learning. Especially, underwater image - enhancement, - fog removal, - segmentation, - noise reduction, - saliency detection and - color constancy under marine environment will be discussed in detail. The cardinal contents of this review are summarized in the below: 
$>$ A relatively comprehensive review of underwater image processing is conducted to categorized off-the-shelf models into seven sub-classes;

$>$ Some publicly accessible underwater-image data sets used in the literatures are presented;

$>$ The main problems and challenges in the underwater vision field are discussed along with some suggestions for future investigation of underwater image processing.

The reminder of this survey is presented as below. Section 2 introduces the representative frameworks on different underwater image processing (UIP) and analysis approaches. Section 3 introduces the designed system at length. Then the challenges and future suggestions are presented in Section 4. Finally, a conclusion will be given in Section 5.

\section{Review of underwater image processing and analysis models}

In the past, various underwater vision systems have been designed to handle and ameliorate the visual quality of underwater images. Generally, underwater image processing (UIP) and analysis frameworks can be classified into seven representative types in terms of the respective target. Figure 1 illustrates the diagram of the underwater image processing and analysis framework. In the following sections, we will describe the details of each individual category.

\subsection{Underwater image enhancement}

Underwater image enhancement aims to improve visual perception and the applicability of an underwater image according to peculiar or specific application occasions. More specifically, the ultimate purpose is to strengthen the visual appearance of the original underwater images so as to emphasize the whole or partial related image characteristics based on the tangible application goal [80]. For instance, disposing of blurred images under a water body condition with serious turbidity becomes clearer or highlighting the underwater image characteristics of the targeted object under a poor lighting condition to satisfy some special analysis purposes.

By modeling the image enhancement as a process of distortion, Liu et al. [3] proposed a reliable image-enhancement method based on adversarial deep networks for underwater images. In [6], Zhang et al. presented a real-time underwater image enhancement procedure via a color constancy-based scheme. They utilized the integration of bilateral as well as trilateral filters to achieve enhancement, which extended the retinex framework for a higher performance. Compared with recent deep learning-based solutions, that approach demonstrates a high performance in both stability and generalization with a minimized computation cost. Through combining an improved retinal algorithm with a neural network, an underwater image enhancement algorithm was developed to improve the reconstruction effect of object edges along with texture details [7]. In [8], He et al. presented an image enhancement model according to the color transfer theory, as well as an efficient pulse-coupled neural network (PCNN) was applied to simultaneously ameliorate the visual appearances in the darker regions, with the aim of improving the contrastive effect in the original underwater image. 


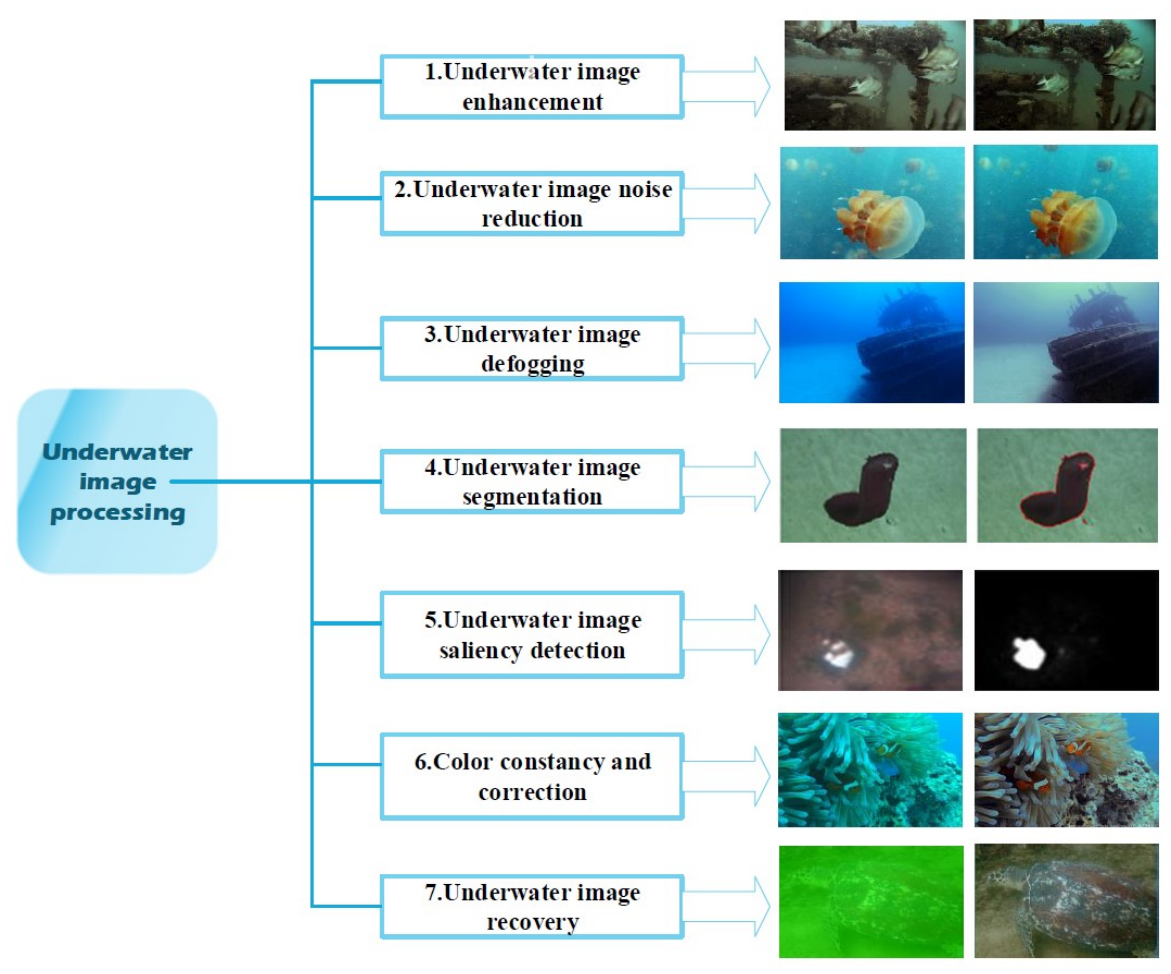

Fig. 1. Diagram of underwater image processing (UIP) and analysis.

Faced with the underwater environment, when light spreads in the water, the effect of absorption and scattering usually leads to negative influences on underwater imaging, such as under-exposure, short-tempered color distortion [77]. To tackle these problems, Fu et al. [9] designed a helpful image enhancement model in terms of correction strategies focusing on the color distortion problem, and then employed a neural network to improve the reflectivity and intensity of illumination for generating the final enhanced underwater image. Lu et al. [10] devised a guided triangular bilateral filter enhancement model to remedy the major reduction with the purpose of solving the image degradation attributed to the color distortion, absorption and scattering of water. This automatic color enhancement algorithm can significantly reduce the noise level and better exposes the dark area in underwater scene. Later, Lu et al. [11] designed a contrastive underwater enhancement framework by exploiting a color correction scheme with a local adaptive filtering for spectral features to recover the distorted color. This framework can generate underwater images with a holistic enhancement while maintaining the local characteristics of the target. In [36], in light of maximum information-retention mechanism, Li et al. exploited a contrast enhancement algorithm for underwater images via restoring their intrinsic visibility. It implied a transcendentally contrastive enhancement procedure in view of histogram distribution to highlight the shadows and brightness of the underwater image. By adapting color balance to the basis of biasness rectification and the mean luminance, Park et al. [43] introduced an effective model for improving the visual effects so as to decrease noises in the enhanced images while intending to keep color fidelity. To adjust the underwater camera images clearer, Li et al. [37] developed an underwater image enhancement framework, with the aid of an adaptive light color channel and a de-noising method to alleviate noise and thus improve low-illumination image to produce clearer visual appearance. In [40], Khan et al. described a restoration enhancement 
approach for degraded underwater images by reducing blurring effects and enhances color and brightness of the underwater images. Recently, Li et al. [60] proposed a reliable underwater image enhancement network (water-net) and an Underwater Image Enhancement Benchmark for promoting underwater vision development.

In the recent past, Liu et al. [67] developed a credible underwater image enhancement framework in accordance with an iterative scheme by generating hybrid image layer propagation. In that depth model, the priors of multiple depth training were integrated into the task. In [68], Hou et al. developed an effective depth-oriented model for underwater image enhancement with the assistance of residual structure estimation and lighting equilibrium. Similar to [71], Zhang et al. [72] presented an underwater image enhancement solution through a color constancy-based scheme, which extended the retinex framework for a higher performance. Compared with recent deep learning-based solutions their approach still demonstrates a high performance in both stability and generalization with a minimized computation cost. More recently, with the assistance of underwater scene prior, a video and image enhancement framework [78] was designed to generate clear underwater frame/video rather than reckoning the parameters of underwater attenuation model.

\subsection{Underwater image noise reduction}

Various noises are commonly emerged in the process of underwater image capturing and transmitting, which are often caused by the camera/sensor equipment and the different severe underwater environments. Due to the influence of image/video acquisition devices, poor illumination quality, serious optical wavelength scattering/absorption and high water turbidity, the noises of the underwater image are always more complex than normal natural images, therefore noise reduction for underwater images is a very important category in underwater image processing. For the sake of ameliorating the visual appearance of underwater images as well as reducing the background noises, many effective noise reduction models for underwater images have been explored in last several decades.

Corrigan et al. [1] presented a mosaicing procedure for underwater videos, which used a time smoothing prior in motion parameters to reduce noise to max the posterior homography assessment calculation method, so as to obtain a small amount texture details. Anwer et al. [2] proposed a prime refraction rectification model, which is able to effectively reduce the noises and eliminate distortions in the acquired underwater image. In the interest of conquering the limitation of the underwater imaging due to absorption and scattering, Zhou, etc. [4] proposed a combinational model on the strength of cosine similarity to tackle the problem of water turbidity of uncertainty.

On the other hand, refraction, uneven homogeneity of light sources and objects' shade are also the fundamental causes of underwater image noises. Therefore, reducing or eliminating their impact has become a primary task for the noise reduction of underwater images. Elnashef and Filin [5] described a model by separating non-refraction sets to reduce the adverse effects of refraction in underwater images. Lu et al. [20] developed a noise reduction framework in terms of self-similarity for underwater noise reduction and super-resolution. In this framework, they proposed a super-resolution algorithm to dispel noise of underwater images with high turbidity, which has indicated that the reconstructed high resolution (HR) image after denoising processing with reasonable noise level can be generated. Sun et al. [42] introduced a rapid underwater image 
enhancement algorithm according to the depth-pixel network framework through employing the skip connection to prevent visual details erosion during the enhancing process.

\subsection{Underwater images defogging}

The excellency of underwater images is seriously under the influence of the underwater light propagation mechanism and imaging devices, such as light absorption, attenuation and backscatter. Light scattering is the main cause of generating blurry and fuzzy image [12, 13, 79], with a goal of guaranteeing the sharpness of the underwater image, it is very important to dispose of such images.

Currently, defogging methods are not effective due to poor understanding of its underlying light transmission mechanisms. Emberton et al. [12] presented an efficient fog-removing algorithm for underwater images, which exploits a type of target estimation with cover light and transmission light to cope with defogging problem. In [13], Skinner and Matthew introduced an underwater image dehazing method to capture the spatial distribution and the angle of images by using light field camera. In this method, each sub-aperture input is initially defogged into two dimensions and then combined together to generate a smooth central view output in the controlled underwater circumstance. In [14], Ancuti et al. designed an underwater image defogging model by correcting contrast and sharpening the white balance to alleviate the defogging effect.

In practice, it is still a challenge for present underwater processing techniques to tackle illumination dispersion and chrominance distortions $[27,71]$. In view of this issue, Chiang et al. [27] proposed a systematic framework to improve the visual quality via applying a dehazing procedure which compensates the decay during the propagation process. In their scheme, the foreground and background in the input are separated. And then an artificial light source is adopted to compensate and enhanced the image in the dehazing procedure. Deng et al. [31] exploited a useful underwater image enhancement approach through combing color removal of the light source with fog removal. By considering the attenuation in various illumination circumstances, the initial image could be defogged with scene depth estimation. In order to enhance the appearance of underwater images, Chiang et al. [34] introduced an effective defogging approach based on dark channel clue, which was used to eliminate the haze effect in the original underwater image. Li et al. [39] designed an efficient framework based on an integrated regression model for underwater image dehazing by integrating chrominance rectification and defogging scheme together to strengthen the contrastive effect of the original input. More recently, Liu et al. [69] designed a new residual architecture that combined the original residual architecture (i.e., domain knowledge) and transmitted information for scene brightness estimation for haze removal with a flexible optimization scheme.

\subsection{Underwater image segmentation}

Underwater image segmentation concentrates on separating the source input into individual and non-overlapping portions, which is a fundamental and critical step for image analysis and understanding. In recent years, plenty of underwater image segmentation models have been investigated.

Although these general image segmentation technologies have been widely exploited, these models may be unsuitable or inapplicable to the underwater environment. This is one of the main reasons that underwater image segmentation technologies lag behind the conventional segmentation. When traditional methods are applied to underwater image processing, the object 
area generally cannot be precisely located, so that the details and shapes of the object will be lost and distorted [41, 45]. To address this deficiency, Chen et al. [41] constructed a new method for underwater target segmentation by modeling the effect of fog haze of underwater image, in which edge level transmission characteristics and regional level significant clues are mixed together with the formula of level set. Based on the statistical property of gray image histogram, Wang et al. [44] proposed an effective fuzzy C-means approach based on particle swarm optimization for underwater optical division. Zhang et al. [45] combined the characteristics of underwater images according to fractal theory, and then proposed an underwater segmentation method assisted by the Brownian random field. Li et al. [46] proposed an efficacious framework to cope with separating large-scale underwater images. In this method, an iterative MapReduce procedure is designed to enhance the efficiency of the algorithm. In accordance with the principle of minimum entropy loss, Wang et al. [50] devised a deep neural network framework in order to divide underwater images into several regions via a gradient optimization algorithm.

The refraction and scattering of light in water affect all aspects of underwater image processing, and also cause serious obstacles to underwater image segmentation. Therefore, it is always a formidable challenge to weaken or even eliminate the adverse effects of light. Based on the level set method, Chen et al. [47] proposed a feature-extraction oriented model through removing background noises and highlighting the outline of underwater objects. This method can preserve the contour of underwater objects during underwater image segmentation. Zhu et al. [48] designed a detection algorithm based on clustering to highlight significant areas in underwater images. On this basis, a statistical active contour approach on the basis of regional level set has proposed to segment underwater images. This model is capable of generating satisfactory effects on underwater image segmentation in terms of efficiency and quality. Rai et al. [49] presented a rapid underwater segmentation approach to separate the object of interest, while the global visual effect was improved via a contrastive histogram flattening algorithm.

\subsection{Underwater image saliency detection}

The human perception system has the congenital capability to efficiently distinguish and perceive salient objects in images or videos $[60,61,76]$. This visual attention mechanism is effective and intelligent by focusing on useful information of the visual scene understanding in natural environments as humans automatically process the areas of interest and selectively ignore the areas not concerned. In the underwater computer vision and image processing domain, saliency detection is intended to equip computers with the ability of human beings to comprehend underwater scenes, which also plays a crucial role in exploring marine resources. Therefore, plenty of underwater saliency detection models have been designed recently.

Because of the uncertainty of underwater environment, it is a challenge to detect the salient objects from underwater images via traditional saliency detection methods. The diversity and complexity of underwater environment has been induced widespread research interest lately. Xu et al. [35] developed a novel approach for underwater target recognition in view of the generalized robust principal component analysis (GRPCA), which could extract the visual feature information from underwater images and was of great significance to the recognition and representation of subordinate images. Shen et al. [51] explored a useful underwater target layered background framework on account of a frog eye visual information perception and processing scheme, which is able to separate salient objects from the image background with object contour. Based on 
underwater image feature extraction and matching, Zhang et al. [52] developed a visual saliency detection model using a dark channel to remove haze. This modal is capable of ameliorating the visibility of underwater images with the aid of dark feature extraction. Chen et al. [53] proposed an effective knowledge-based weighted integration strategy for salient region detection by using underwater target extraction, which was propitious to the dispersibility of the illumination characteristics. Later, Chen et al. [54] also devised an underwater saliency detection model by considering both 2D and 3D depth clues together. In [55], Li et al. exploited an underwater image saliency detection framework in light of foreground extraction to solve the problems evoked by the low quality image with low contrast. By locating the geometric center using the Harris corner detection operator, a simple linear iterative clustering based procedure was devised via protruding the foreground object and weakening the background areas for underwater image saliency detection [56]. Chen et al. [57] designed a salient object detection approach with aid of a monocular vision sensor to identify underwater objects. In this approach, background noises were removed to refine the detection accuracy in underwater scenes. In [58], Chen et al. devised an efficient model by exploiting a super-resolution generative adversarial network to improve the visual effect of image for detecting and recognizing underwater objects. Sun et al. [59] proposed a convolutional neural network based model for underwater target recognition. Among their model, a weighted probability decision mechanism is applied to distinguish objects from low-contrast underwater images. In [62], Jian et al. developed a saliency-detection method through combining Quaternionic features and object contrastive cues. Experiments performed on the partial of the publicly available underwater image database [63] demonstrated the effectiveness of the method. With the aid of removing color aberration and enhancing contrast of underwater images, Zhang et al. [70] presented an underwater image saliency detection framework by using deformable convolutional networks. Softmax was applied to regress the feature information of each salient object so that the spatial location could be estimated.

\subsection{Color constancy and color correction for underwater images}

Color constancy is a type of perceptual constancy, which tends to remain relatively stable even for individual perception of familiar objects under different changes in illumination, lighting intensity and other conditions. Therefore, keeping color constancy is helpful for vision-based applications and is an important topic of underwater image processing.

Since underwater vision is seriously affected by the optical wave attenuation and dispersion in water medium, the application and generalization of visual models are normally impaired. Li et al. [22] designed a supervised learning based color conversion algorithm for rectifying distortion of underwater images, which was assisted by a cyclically consistent network by relaxing the requirement for the training sets. To tackle the instability of the underwater color reconstruction model, Akkaynak and Treibitz [23] described a color correction model by controlling direct transmission coefficients of the underwater color reconstruction. To solve the problem of the local variable underwater scene, Codruta et al. [24] exploited a framework to utilize color conversion and adjust color correction locally via the light attenuation level function of red channel estimation. And then, they applied the dark channel prior inversion to recover chrominance compensation. In [25], Nomura et al. depicted an effective color correction approach for underwater images in the basis of grayscale linear regression to capture different exposure time for color correction in underwater scene. To address the color conversion problem, Zhang et al. developed a helpful 
underwater image color correction framework in accordance with the retinex algorithm via light adjustment as well as gamma correction [28]. Considering the absorption of various wavelengths often leads to color distortion in practice, $\mathrm{Li}$ and Cavallaro [29] proposed a color restoration method by compensating color wastage caused by the scene-to-camera interval so as to estimate the non-uniform background illumination [74] in underwater scene, which contributes to the progress of underwater vision

\subsection{Underwater image recovery/restoration}

The purpose of underwater image restoration is the process of reconstructing or recovering degraded images caused by the adverse factors in the complex underwater environment, including camera and object relative motion, the optical imaging mechanism of underwater scattering, turbulence, distortion, spectral absorption and attenuation, etc.

To eliminate the interference of medium light transmission radiation, Barros et al. [15] presented an efficient method to restore details of underwater images with aid of a light propagation physical model. In this model, they aimed to reduce the attenuation and degradation of the backward scattering field since scattering and water fluidity frequently cause severe influences on underwater imaging. Meanwhile, Chen et al. [16] established a degradation approach through estimating the turbulent degradation transfer factors during image recovery and reconstruction, the effect of the final outputs can be improved. Different from these underwater image recovery algorithms based on physical models which contain several parameters, a biologically inspired image-restoration method is proposed in [17]. Halimi et al. [18] developed an efficient strategy to enhance the quality of underwater images by jointly reconstructing depth and reflectivity of the original image via maximum marginal likelihood estimation. In [19], Ancuti et al. proposed an effective framework to strengthen the underwater visibility, which is in accordance with weight graphs to distinguish the areas with poor visibility so as to relieve the defects existing in the degraded input image. Based on polarimetric imaging, $\mathrm{Hu}$ et al. [21] developed an underwater image recovery method via estimating the backscatter intensity at different spatial positions. Although polarization mechanism can enhance visual quality in water medium, these existing models are prone to facing problems in obtaining the true distribution of polarization. In order to overcome the issue, Tian et al. [26] proposed a combined framework with aperture with polarimetric imaging to calculate radiation of underwater targets. To address the limitations of the priority-based approach, Wang et al. [30] proposed a transmission-estimation model by employing cross-layer connection to eliminate underwater artifacts as well to recover textural characteristics. In [32], Barbosa et al. also proposed a deep neural image-recovery method to improve the visual effect of underwater images via preserving local characteristics (e.g. edges and contours) of the images. To conquer the limitations of the complexity of underwater environment, Zhang and Peng [33] proposed an underwater medium transmission method via modeling the characteristics of underwater imaging and light source for image restoration. By transforming the underwater propagation mechanism into a transmission model, Wang et al. [38] designed a maximum attenuation identification framework through extracting the depth map from the degraded underwater image to realize underwater image restoration. According to the expansion and correction of dark channel clue, Li et al. [65] developed an underwater image recovery framework based on chrominance channel defogging to solve the problem that the partially recovered image area is too dark or too bright. In [66], Li et al. also exploited an underwater image recovery model 
by estimating the global background light to enhance the brightness as well as visibility of underwater images. Recently, Fan et al. [73] presented a flat refraction model for underwater optical imaging to describe the geometric relation between the refracted image and the actual object, which can rebuild underwater 3-D shapes in laser triangulation and photometric stereo and thus can effectively correct the distortion in underwater 3D reconstruction.

\section{Data sets for underwater image processing and analysis}

Because of underwater image processing is a newly emerging research direction in recent years, there are only a few data sets dedicated to underwater computer vision [63]. The fundamental reasons for this are listed below:

1) The scientific research of underwater image processing started relatively late, so the relevant underwater image data sets have not been paid enough attention.

2) Although academic researchers have currently noticed the importance of the underwater image database, it takes time to develop these kinds of dataset owing to the complicacy of the underwater circumstances and the high workload.

3) Owing to the diversity of underwater environment, the ground truths of various underwater images are very difficult to obtain and label manually.

Table 1 summarized some existing typical databases, which can be publicly available for underwater image processing and analysis in detail. 
Table 1 . Some publicly accessible databases for underwater image processing.

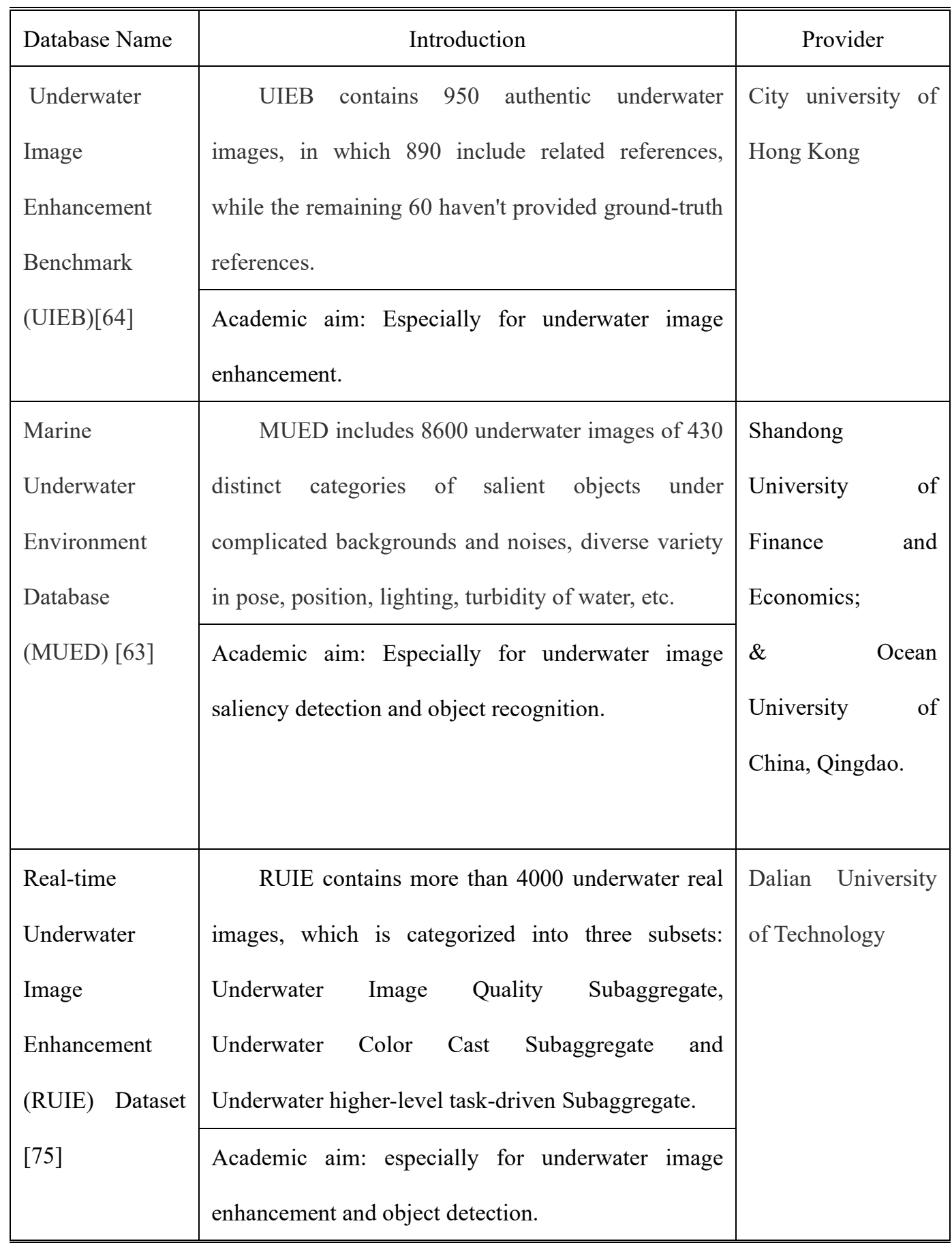


In particular, Underwater Image Enhancement Benchmark (UIEB) [64] contains a couple of corresponding subclasses with 890 pairs of original underwater images and reference images on a one-for-one basis, as shown in Figure 2.
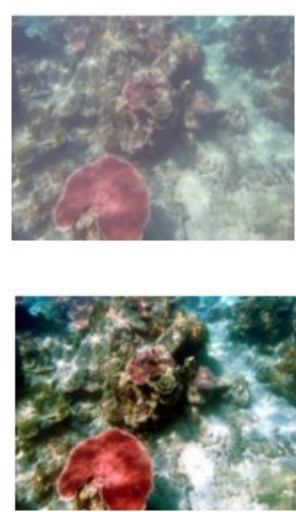

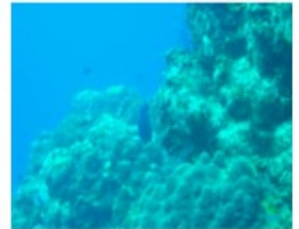

(a)
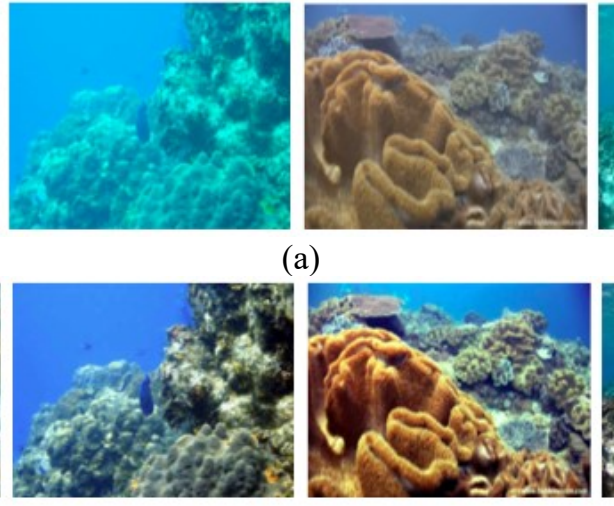

(b)
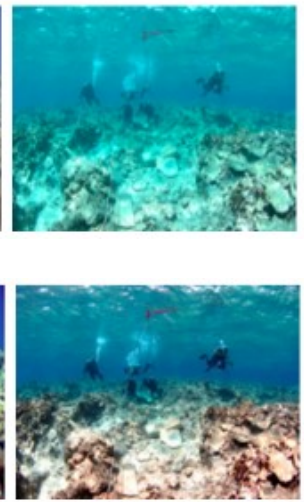

Fig. 3. Some examples from UIEB [64] with a couple of subclasses : (a) original underwater images, (b) corresponding reference images.

Figure 3 shows some samples of underwater images with high turbidity, uneven illumination, monotonous color, and complicated underwater-background from MUED [63], which seriously affect the integrity and availability of underwater image in practical application.

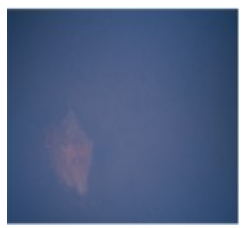

(a)

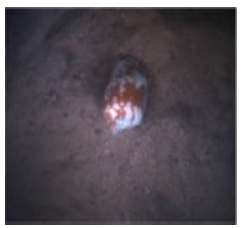

(b)

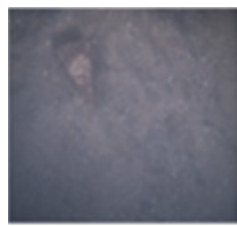

(c)

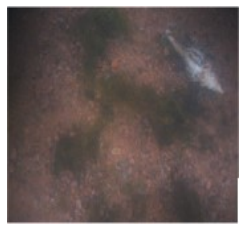

(d)

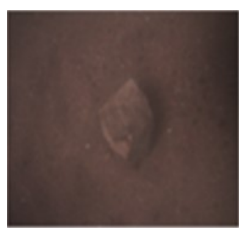

(e)

Fig. 3. Some samples of adverse factors in the marine environment for underwater vision applications [63]. (a) Water with high turbidity, (b) uneven illumination, (c) low contrast, (d) complicated underwater-background, and (e) monotonous colour.

Figure 4 displays some images from Real-time Underwater Image Enhancement (RUIE) Dataset which is constructed via an underwater optical imaging and capturing system [75]. RUIE includes a triple of subclasses of underwater images with Underwater Image Quality Subaggregate, Underwater Color Cast Subaggregate and Underwater higher-level task-driven Subaggregate.

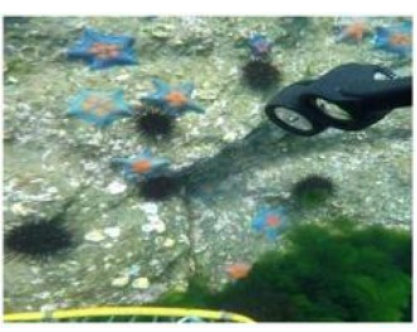

(a)

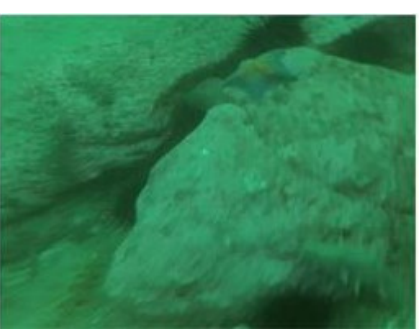

(b)

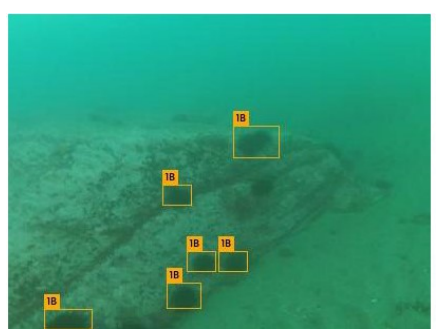

(c)

Fig. 4. Some images from RUIE Dataset [75] with a triple of subclasses of underwater images: (a) Underwater Image Quality Subaggregate, (b) Underwater Color Cast Subaggregate, (c) Underwater higher-level task-driven Subaggregate. 


\section{Challenges and future suggestions}

The main purpose of this review is to summarize recent research, identify the problems and challenges in the related underwater image field and then intend to provide some insights and suggestions for the future direction. The following is a brief summary of the open problems and challenges, which will be conducive to the promotion of the progress of underwater image processing and thus attract attention to researchers in signal processing and computational intelligence.

$>$ Turbidity of underwater quality, light refraction, absorption and scattering in underwater environments are still the core factors affecting the effect of underwater image quality. Currently, most of the existing models are focused on one single factor listed above. In future research, multiple influencing factors can be considered comprehensively to enhance the quality of underwater images.

$>$ Low-contrast objects with complicated seabed scene, changeable underwater environmental illumination conditions, and severe turbidity resulting in blurred images and monochrome underwater objects without abundant color information jointly constitute a major obstacle to promotion on underwater saliency detection and object recognition.

$>$ Although the captured underwater image influenced by the imaging equipment itself, the interference of the absorption and scattering of the optical wave propagation in turbulent underwater also frequently leads to image distortion with a distinct difference between the final image acquisition and the real scene. Light-propagation based the physical model is still worth further investigating thoroughly to address the intrinsic effects of these factors, which likely needs a long time to grope for underwater computer-vision research.

$>$ Research on deep-learning methods for underwater image processing is only at the early stage. Currently, the lack of large-scale underwater database limits and impedes the deep-learning based models on the application to underwater image processing. As more underwater image datasets with labeled ground truth are released availably, deep-learning based frameworks for underwater image processing will be increased tremendously in the future.

\section{Conclusions}

In this article, we have summarized a brief review of recent research related to the underwater image processing and analysis. Previously representative methods are divided into seven distinct categories: Underwater image, enhancement, noise reduction, defogging, segmentation, saliency detection, color constancy and recovery/restoration. Furthermore, we have also presented the datasets applied in underwater image processing. Finally, we systematically analyze the challenges in the underwater image processing domain and provide some suggestions for the future development of underwater image processing.

\section{Acknowledgment}

This work was supported by National Natural Science Foundation of China (NSFC) (61976123, 61601427, 61876098); the Taishan Young Scholars Program of Shandong Province; and Royal Society - K. C. Wong International Fellowship (NIF $\backslash R 1 \backslash 180909)$. 


\section{REFERENCES}

[1] David Corrigan, Ken Sooknanan, Jennifer Doyle, Colm Lordan, and Anil Kokaram, "A Low-Complexity Mosaicing Algorithm for Stock Assessment of Seabed-Burrowing Species." IEEE Journal of Oceanic Engineering, 44.2 (2018): 386-400.

[2] Atif Anwer, Syed Saad Azhar Ali, Amjad Khan, Fabrice Meriaudeau, "Underwater 3-d scene reconstruction using kinect v2 based on physical models for refraction and time of flight correction." IEEE Access, 5 (2017): 15960-15970.

[3] Peng Liu, Guoyu Wang, Hao Qi, Chufeng Zhang, Haiyong Zheng, Zhibin Yu, "Underwater Image Enhancement with a Deep Residual Framework." IEEE Access, 7 (2019): 94614-94629.

[4] Lina Zhou, Yin Xiao ,and Wen Chen, "Imaging through turbid media with vague concentrations based on cosine similarity and convolutional neural network." IEEE Photonics Journal, 11.4 (2019): $1-15$.

[5] Bashar Elnashef, Sagi Filin, "Direct linear and refraction-invariant pose estimation and calibration model for underwater imaging”, ISPRS Journal of Photogrammetry and Remote Sensing, 154 (2019) 259-271.

[6] Zhang S., Wang, T., Dong, J., \& Yu, H. Underwater image enhancement via extended multi-scale retinex. Neurocomputing (2017), 245, 1-9.

[7] Yujie Li, Chunyan Ma, Tingting Zhang, Jianru Li, Zongyuan Ge, Yun Li, And Seiichi Serikawa,

"Underwater Image High Definition Display Using the Multilayer Perceptron and Color Feature-Based SRCNN." IEEE Access, 7 (2019): 83721-83728

[8] Kangjian He, Ruxin Wang, Dapeng Tao, Jun Cheng, Weifeng Liu , "Color transfer pulse-coupled neural networks for underwater robotic visual systems." IEEE Access, 6 (2018): 32850-32860.

[9] Xueyang Fu, Peixian Zhuang, Yue Huang, Yinghao Liao, Xiao-Ping Zhang, Xinghao Ding, "A retinex-based enhancing approach for single underwater image." 2014 IEEE International Conference on Image Processing (ICIP). IEEE, (2014) : 4572-4576.

[10] Huimin Lu, Yujie Li, and Seiichi Serikawa. "Underwater image enhancement using guided trigonometric bilateral filter and fast automatic color correction." 2013 IEEE International Conference on Image Processing. IEEE, (2013) : 3412-3416.

[11] Huimin Lu, Yujie Li, Lifeng Zhang, and Seiichi Serikawa, "Contrast enhancement for images in turbid water." Journal of the Optical Society of America A, 32.5 (2015): 886-893.

[12] Emberton Simon, Lars Chittka, and Andrea Cavallaro. "Hierarchical rank-based veiling light estimation for underwater dehazing." (2015).

[13] Katherine A. Skinner, and Johnson-Roberson Matthew. "Underwater image dehazing with a light field camera." Proceedings of the IEEE Conference on Computer Vision and Pattern Recognition Workshops.( 2017):62-69.

[14] Codruta O. Ancuti, Cosmin Ancuti, Christophe De Vleeschouwer, and Philippe Bekaert, "Color balance and fusion for underwater image enhancement." IEEE Transactions on Image Processing, 27.1 (2017): 379-393.

[15] Wagner Barros, Erickson . Nascimento, Walysson V. Barbosa, Mario F.M. Campos, "Single-shot underwater image restoration: A visual quality-aware method based on light propagation model." Journal of Visual Communication and Image Representation ,55 (2018): 363-373.

[16] Yuzhang Chen, Zhangfan Zeng, and Yongcai Pan. "A New Degradation Model for Imaging in Natural Water and Validation Through Image Recovery." IEEE Access ,7 (2019): 123244-123254. 
[17] C. S'anchez-Ferreira, L. S. Coelho, H. V. H. Ayala, M.C.Q. Farias, C. H. Llanos, "Bio-inspired Optimization Algorithms for Real Underwater Image Restoration", Signal Processing: Image Communication, (2019).

[18] Abderrahim Halimi, Aurora Maccarone, Aongus McCarthy, Steve McLaughlin, and Gerald S. Buller, "Object depth profile and reflectivity restoration from sparse single-photon data acquired in underwater environments." IEEE Transactions on Computational Imaging, 3.3 (2017): 472-484.

[19] Codruta Orniana Ancuti,Cosmin Ancuti,Tom Haber,and Philippe Bekaert "Fusion-based restoration of the underwater images." 2011 18th IEEE International Conference on Image Processing. IEEE, (2011): 1557-1560.

[20] Huimin Lu, Yujie Li, Shota Nakashima, Hyongseop Kim, And Seiichi Serikawa, "Underwater image super-resolution by descattering and fusion." IEEE Access, 5 (2017): 670-679.

[21] Haofeng $\mathrm{Hu}$, Lin Zhao,Xiaobo Li ,Hui Wang, and Tiegen Liu, "Underwater image recovery under the nonuniform optical field based on polarimetric imaging." IEEE Photonics Journal ,10.1 (2018): 1-9. [22] Chongyi Li, Chunle Guo, and Jichang Guo, Emerging from water: Underwater image color correction based on weakly supervised color transfer, IEEE Signal Processing Letters, vol. 25, no. 3, pp. 323-327, 2018.

[23] Akkaynak, Derya, and Tali Treibitz. "A revised underwater image formation model." Proceedings of the IEEE Conference on Computer Vision and Pattern Recognition. 2018 : 6723-6732.

[24] Codruta O. Ancuti, Cosmin Ancuti, Christophe De Vleeschouwer, Rafael Garcia, "Locally adaptive color correction for underwater image dehazing and matching." Proceedings of the IEEE Conference on Computer Vision and Pattern Recognition Workshops. 2017: 1-9.

[25] Nomura Kohei, Daisuke Sugimura, and Takayuki Hamamoto. "Underwater image color correction using exposure-bracketing imaging." IEEE Signal Processing Letters, 25.6 (2018): 893-897.

[26] Yu Tian, Bin Liu, Xinyan Su, Lipeng Wang, Ke Li, "Underwater Imaging Based on LF and Polarization." IEEE Photonics Journal, 11.1 (2019): 1-9.

[27] John Y. Chiang and Ying-Ching Chen, "Underwater Image Enhancement by Wavelength Compensation and Dehazing”,IEEE Transactions on Image Processing ,21.4 (2011): 1756-1769.

[28] Wenhao Zhang, Ge Li, and Zhenqiang Ying. "A new underwater image enhancing method via color correction and illumination adjustment." 2017 IEEE Visual Communications and Image Processing (VCIP). IEEE, $2017: 1-4$.

[29] Chau Yi Li, and Andrea Cavallaro. "Background light estimation for depth-dependent underwater image restoration." 2018 25th IEEE International Conference on Image Processing (ICIP). IEEE, 2018 : 1528-1532.

[30] Keyan Wang, Yan Hu , Jun Chen, Xianyun Wu, Xi Zhao and Yunsong Li, "Underwater Image Restoration Based on a Parallel Convolutional Neural Network." Remote Sensing,11.13 (2019): 1591.

[31] Xiangyu Deng, Huigang Wang, and Xing Liu. "Underwater Image Enhancement Based on Removing Light Source Color and Dehazing." IEEE Access, 7 (2019): 114297-114309.

[32] Walysson V. Barbosa, Henrique G. B. Amaral, Thiago L. Rocha, Erickson R. Nascimento, "Visual-Quality-Driven Learning for Underwater Vision Enhancement." 2018 25th IEEE International Conference on Image Processing (ICIP). IEEE, 2018 : 3933-3937.

[33] Mohua Zhang, and Jianhua Peng. "Underwater Image Restoration Based on a New Underwater Image Formation Model." IEEE Access, 6 (2018): 58634-58644.

[34] JohnY Chiang, YingChing Chen, and YungFu Chen. "Underwater image enhancement: using wavelength compensation and image dehazing (WCID)." International Conference on Advanced 
Concepts for Intelligent Vision Systems. Springer, Berlin, Heidelberg, 2011 : 372-383.

[35] Jian Xu, Pengfei Bi , Xue Du, Juan Li, Dong Chen, "Generalized Robust PCA: A New Distance Metric Method for Underwater Target Recognition." IEEE Access, 7 (2019): 51952-51964.

[36] Chongyi Li, Jichang Guo, Runmin Cong, Yanwei Pang, Bo Wang, Underwater image enhancement by dehazing with minimum information loss and histogram distribution prior, IEEE Transactions on Image Processing, vol.25, no.12, pp.5664-5677, 2016.

[37] Yujie Li , Jianru Li, Yun Li, Hyoungseop Kim, And Seiichi Serikawa, "Low-Light Underwater Image Enhancement for Deep-Sea Tripod." IEEE Access ,7 (2019): 44080-44086.

[38] Nan Wang, Haiyong Zheng, and Bing Zheng. "Underwater image restoration via maximum attenuation identification." IEEE Access, 5 (2017): 18941-18952.

[39] Chongyi Li, Jichang Guo, Chunle Guo, Runmin Cong, and Jiachang Gong, A hybrid method for underwater image correction, Pattern Recognition Letters, vol. 94, pp. 62-67, 2017.

[40] Amjad Khan, Syed Saad Azhar Ali, Atif Anwer, Syed Hasan Adil, And Fabrice Meriaudeau,

"Subsea Pipeline Corrosion Estimation by Restoring and Enhancing Degraded Underwater Images." IEEE Access, 6 (2018): 40585-40601.

[41] Zhe Chen, Yang Sun, Yupeng Gu, Huibin Wang, Hao Qian, And Hao Zheng, "Underwater Object Segmentation Integrating Transmission and Saliency Features”,IEEE Access, Volume 7, 2019

[42] Xin Sun, Lipeng Liu, Qiong Li, Junyu Dong, Estanislau Lima, Ruiying Yin, "Deep pixel-to-pixel network for underwater image enhancement and restoration." IET Image Processing,13.3 (2018): 469-474.

[43] Park Dubok, David K. Han, and Hanseok Ko. "Enhancing Underwater Color Images via Optical Imaging Model and Non-Local Means Denoising." IEICE TRANSACTIONS on Information and Systems, 100.7 (2017): 1475-1483.

[44] Shilong Wang, Yuru $\mathrm{Xu}$, and Yongjie Pang. "A fast underwater optical image segmentation algorithm based on a histogram weighted fuzzy C-means improved by PSO." Journal of Marine Science and Application, 10.1 (2011): 70-75.

[45] Tiedong Zhang, Lei Wan, Zaibai Qin, Lu Yu, "A method of underwater image segmentation based on discrete Fractional Brownian Random Field." 2008 3rd IEEE Conference on Industrial Electronics and Applications, $2008: 2507-2511$.

[46] Xiu Li, Jingdong Song, Fan Zhang, Xiaogang Ouyang, Samee U. Khan, "MapReduce-based fast fuzzy c-means algorithm for large-scale underwater image segmentation." Future Generation Computer Systems, 65 (2016): 90-101.

[47] Zhe Chen, Zhen Zhang, Yang Bu, Fengzhao Dai, Tanghuai Fan and Huibin Wang, "Underwater object segmentation based on optical features." Sensors, 18.1 (2018): 196.

[48] Yue Zhu,Baochen Hao, Baohua Jiang, RuiNian, Bo He, Xinmin Ren, "Underwater image segmentation with co-saliency detection and local statistical active contour model." OCEANS 2017-Aberdeen. IEEE, $2017: 1-5$.

[49] Kumar Rai, Rajesh, Puran Gour, and Balvant Singh. "Underwater image segmentation using clahe enhancement and thresholding." International Journal of Emerging Technology and Advanced Engineering, 2.1 (2012): 118-123.

[50] Bo Wang, Lei Wan, and Ye Li. "Saliency motivated pulse coupled neural network for underwater laser image segmentation." Journal of Shanghai Jiaotong University (Science), 21.3 (2016): 289-296.

[51] Jie Shen, Tanghuai Fan,Min Tang, Qian Zhang, Zhen Sun, and Fengchen Huang, "A biological hierarchical model based underwater moving object detection." Computational and mathematical 
methods in medicine, (2014).

[52] Lunjuan Zhang, Bo He, Yan Song ,And Tianhong Yan, "Underwater image feature extraction and matching based on visual saliency detection." IEEE OCEANS 2016-Shanghai, 2016: 1-4.

[53] Zhe Chen, Huibin Wang, Lizhong Xu, Jie Shen, "Visual-adaptation-mechanism based underwater object extraction." Optics \& Laser Technology, 56 (2014): 119-130.

[54] Zhe Chen, Hongmin Gao, Zhen Zhang, Helen Zhou, Xun Wang, Yan Tian, "Underwater salient object detection by combining 2D and 3D visual features." Neurocomputing, (2019).

[55] Xiu Li, Jing Hao, Min Shang, Zhixiong Yang, "Saliency segmentation and foreground extraction of underwater image based on localization." OCEANS 2016-Shanghai. IEEE, 2016: 1-4.

[56] Li Mou, Xuewu Zhang, Jingjing Zhang, Xiaohai Shen, Xiaolong Xu, "Saliency Detection of Underwater Target Based on Spatial Probability." 2017 International Conference on Computer Systems, Electronics and Control (ICCSEC). IEEE, 2017 : 630-632.

[57] Zhe Chen, Zhen Zhang, Fengzhao Dai, Yang Bu and Huibin Wang, "Monocular vision-based underwater object detection." Sensors ,17.8 (2017): 1784.

[58] Zhengyu Chen, Tongtong Zhao, Na Cheng, Xundong Sun, Xianping Fu, "Towards Underwater Object Recognition Based on Supervised Learning." 2018 OCEANS-MTS/IEEE Kobe Techno-Oceans (OTO). IEEE, 2018 : 1-4.

[59] Xin Sun, Junyu Shi, Lipeng Liu, Junyu Dong, Claudia Plant, Xinhua Wang, Huiyu Zhou, "Transferring deep knowledge for object recognition in Low-quality underwater videos." Neurocomputing, 275 (2018): 897-908.

[60] Muwei Jian, Kin-Man Lam, Junyu Dong, Linlin Shen, "Visual-patch-attention -aware Saliency Detection", IEEE Trans. on Cybernetics, 45 (2015), 1575-1586.

[61] Muwei Jian, Wenyin Zhang, Hui Yu, Chaoran Cui, Xiushan Nie, Huaxiang Zhang, Yilong Yin, "Saliency detection based on directional patches extraction and principal local color contrast." Journal of Visual Communication and Image Representation 57 (2018): 1-11.

[62] Muwei Jian, Qiang Qi, Junyu Dong, Yilong Yin, Kin-Man Lam, "Integrating QDWD with pattern distinctness and local contrast for underwater saliency detection." Journal of visual communication and image representation, 53 (2018): 31-41.

[63] Muwei Jian, Qiang Qi, Hui Yu, Junyu Dong, Chaoran Cui, Xiushan Nie, Huaxiang Zhang, Yilong Yin, Kin-Man Lam, "The extended marine underwater environment database and baseline evaluations." Applied Soft Computing, 80 (2019): 425-437.

[64] Chongyi Li, Chunle Guo, Wenqi Ren, Runmin Cong, Junhui Hou, Sam Kwong, and Dacheng Tao, An underwater image enhancement benchmark dataset and beyond, IEEE Transactions on Image Processing, vol.29, pp. 4376-4389, 2019.

[65] Chongyi Li, Jichang Guo, Yanwei Pang, Shanji Chen, Jian Wang, "Single underwater image restoration by blue-green channels dehazing and red channel correction." 2016 IEEE International Conference on Acoustics, Speech and Signal Processing (ICASSP). IEEE, 2016: 1731-1735.

[66] Chongyi Li, Jichang Guo, Shanji Chen, Yibin Tang, Yanwei Pang, Jian Wang, "Underwater image restoration based on minimum information loss principle and optical properties of underwater imaging." 2016 IEEE International Conference on Image Processing (ICIP). IEEE, 2016: 1993-1997.

[67] Risheng Liu, Minjun Hou, Jinyuan Liu, Xin Fan, Zhongxuan Luo, "Compounded Layer-Prior Unrolling: A Unified Transmission-Based Image Enhancement Framework." 2019 IEEE International Conference on Multimedia and Expo (ICME). IEEE, 2019 : 538-543.

[68] Minjun Hou, Risheng Liu, Xin Fan, Zhongxuan Luo, "Joint residual learning for underwater 
image enhancement." 2018 25th IEEE International Conference on Image Processing (ICIP). IEEE, $2018:$ 4043-4047.

[69] Risheng Liu, Xin Fan, Minjun Hou, Zhiying Jiang, Zhongxuan Luo, and Lei Zhang, "Learning aggregated transmission propagation networks for haze removal and beyond." IEEE transactions on neural networks and learning systems, (2018).

[70] Dong Zhang, Lan Li, Zizhong Zhu, Shangang Jin, Weizhe Gao, Ce Li, "Object Detection Algorithm Based on Deformable Convolutional Networks for Underwater Images." 2019 2nd China Symposium on Cognitive Computing and Hybrid Intelligence (CCHI). IEEE, 2019 : 274-279.

[71] Muwei Jian, Kin-Man Lam, Junyu Dong, Illumination-insensitive Texture Discrimination Based on Illumination Compensation and Enhancement, Information Sciences, 269, pp. 60-72, 2014.

[72] S. Zhang, T. Wang, J. Dong, H. Yu, Underwater image enhancement via extended multi-scale retinex. Neurocomputing, 245, 1-9, 2017.

[73] H. Fan, L. Qi, J., H. Yu, Refractive laser triangulation and photometric stereo in underwater environment. Optical Engineering, 56(11): 113101,2017.

[74] Muwei Jian, et al., Comprehensive Assessment of Non-uniform Illumination for 3D Heightmap Reconstruction in Outdoor Environments, Computers in Industry, 99, 110-118, 2018.

[75] Risheng Liu, Xin Fan, Ming Zhu, Minjun Hou, and Zhongxuan Luo, "Real-world Underwater Enhancement: Challenges, Benchmarks, and Solutions under Natural Light." IEEE Transactions on Circuits and Systems for Video Technology, 2020.

[76] Chongyi Li, Runmin Cong, Junhui Hou, Sanyi Zhang, Yue Qian, and Sam Kwong, Nested network with two-stream pyramid for salient object detection in optical remote sensing images, IEEE Transactions on Geoscience and Remote Sensing, vol. 57, no. 11, pp. 9156-9166, 2019.

[77] Chunle Guo, Chongyi Li, Jichang Guo, Runmin Cong, Huazhu Fu, and Ping Han, Hierarchical features driven residual learning for depth map super-resolution, IEEE Transactions on Image Processing, vol. 28, no. 5, pp. 2545-2557, 2019.

[78] Chongyi Li, Saeed Anwar, and Fatih Porikli, Underwater scene prior inspired deep underwater image and video enhancement, Pattern Recognition, vol. 98, 2020.

[79] Chongyi Li, Chunle Guo, Jichang Guo, Ping Han, Huazhu Fu, and Runmin Cong, PDR-Net: Perception-inspired single image dehazing network with refinement, IEEE Transactions on Multimedia, vol.22, no.3, pp.704-716, 2020.

[80] Miao Yang, Ke Hu, YixiangDu, Zhiqiang Wei, Zhibin Sheng,Jintong Hu, Underwater image enhancement based on conditional generative adversarial network, Signal Processing: Image Communication, Volume 81, February 2020, 115723. 